\title{
The right partner
}

\author{
For the development, application and dissemination of high-impact methods, \\ interdisciplinary collaboration between experts is vital.
}

The image of the scientist as the rarefied solitary thinker, as most practicing scientists recognize, is a cliché and a largely incorrect one at that. Research is most typically an interactive activity and, in biology, one that occurs in ever larger and more diverse groups. What constitutes a well-functioning and creative biological research team? And what is the role of collaboration, particularly interdisciplinary collaboration, in achieving research goals?

There is no shortage of studies showing a correlation between collaboration and citations of the resulting research. Perhaps as a result, funding bodies and institutions are increasingly pushing the value of collaboration, particularly of the interdisciplinary sort. This has even become a field of research in its own right, with an annual Science of Team Science conference. At the same time, the scientific community continues to recognize one or a few individuals for their accomplishments. Prestigious prizes are given to very few scientists and institutions compete over recognized stars.

Interdisciplinarity is particularly vital in the development of biomedical research methods. By its very nature, methods development often requires expertise in subjects quite removed from the biological objects of study. Development of microscopy methods may require intimate knowledge of physics, for instance; a background in mathematics and software coding is important for developing analysis algorithms and software; and knowledge of organic synthesis techniques is necessary for creating chemical-based sensors and probes. But high-impact biological methods development requires more than technical excellence. Development should be guided by need, with the performance of the method honed on an application that is significant for biology.

Although the importance of visionary individuals is undeniable, such work is most typically done in interdisciplinary teams. Because it can be difficult for a single researcher to recruit and manage a strong interdisciplinary team, interlaboratory collaborations often provide invaluable missing expertise to a project.

An examination of papers published in Nature Methods suggests that the number of authors on a paper is predictive of whether it falls in the top or bottom quartile in terms of the numbers of citations per year, although we note that these data have not been statistically analyzed and that citations may reflect properties of a paper other than scientific value. Furthermore, for papers with four or fewer authors, those with all authors in the same department and institute are cited less frequently than those with authors in different departments or institutions. Conversely, for papers with five or more authors, the fraction of highly cited papers increases for those with authors in different institutions.

Indeed, the stories behind many Nature Methods papers tell of interdisciplinary collaboration. Computer scientist Badrinath Roysam, for example, was a perfect collaborator for biologist Michel Cayouette when he sought an algorithm to predict cell differentiation. For two papers in this issue (p. 153 and p. 171), engineer Hang Lu sent one lab member to work with fly biologists and another to scientists studying the behavior of worms. And it took collaboration between developmental biologist Erik Jorgensen and physicist Stefan Hell to place super-resolution imaging in the context of cellular ultrastructure in an organism.

The US National Institutes of Health and other funding agencies have recognized that unanticipated opportunities for interdisciplinary collaboration can arise after a project has been funded and have established programs to support such collaborations with supplements to existing grants. Funding is awarded on a competitive basis, and interest from researchers has been high. Decisions are made by program staff with a quick turnaround so that funds can be obtained without delay. Researchers should also not overlook the positive effect that even informal collaborations may have on obtaining funding in the first place.

Of course, collaboration does not come without difficulties. A problem unique to interdisciplinary projects is that proper credit may not be given to researchers providing crucial technical expertise. Some journals, including Nature Research journals, now implement author contribution statements to counteract this problem. A related issue, one we encounter frequently, is when collaborative groups attempt to publish biological results entirely separately from the methodology itself. This can yield a paper that does not sufficiently probe the potential of a method for gaining biological insight.

Problems notwithstanding, the value of collaboration for methods development is clear. Funders, institutional officers and even conference organizers should continue to promote these opportunities. 